\title{
A Fast Algorithm to Calculate Ultrasound Pressure Fields From Single-Element Transducers
}

\author{
LIN XIN YAO, JAMES A. ZAGZEBSKI, AND EVAN J. BOOTE
}

\begin{abstract}
Computations of the pressure field of ultrasound transducers generally are done by numerically integrating a function that represents contributions from monopole sources distributed over the surface of the transducer. The function is a sinusoid, with an envelope that varies smoothly over the limits of integration. These computations can be speeded up by expressing the slowly changing envelope of the integrand as a polynomial, and thus converting the integral into one that can be solved in closed form. This accelerates the computations by a factor of about 10 or more without compromising accuracy.
\end{abstract}

\section{INTRODUCTION}

$I^{N}$ N MANY AREAS of medical ultrasound research, it is necessary to quantify the pressure field of pulsed, focused transducers [1]-[3]. A common approach is to evaluate the Rayleigh surface integral [4] at each field point, where the integration is done over the active area of the transducer. This approach is rigorous for a uniform plane piston radiator, but for focused transducers, the result is considered an approximation because secondary diffraction is not taken into account [5]. However, the integral provides accurate results for focused transducers as long as the radius of curvature is large compared to the acoustic wavelength, [5], [6]. One difficulty with these computations is that large CPU times are required, even on a main frame computer, because they generally involve twodimension numerical integrations of complex exponential functions. For a disk or spherical shell focused transducer the double integral over the transducer surface can be reduced to a single integral [6], [7] that is solved numerically. Even with this improvement Gaussian quadrature (GQ) integration techniques used thus far in our laboratory [7], [8] require extensive CPU time, especially where large volumes, say greater than 27 cubic centimeters, and broad-band pulses are involved.

Substantial time savings are realized using a Taylor series approximation, expansion being done with respect to the ultrasound frequency [9], [10]. For each field point only one numerical integration is done to calculate Taylor series coefficients. The pressure at that point for all fre-

Manuscript received August 5, 1988; revised and accepted December 23, 1988. This work was supported in part by Grant RO1-CA25634 and RO1-CA39224 from the National Institutes of Health and by Radiation Measurements Incorporated.

L. Yao and J.A. Zagzebski are with the Department of Medical Physics, University of Wisconsin, 1300 University Avenue, Room 1530, Madison, WI 53706

E. J. Boote is with the Department of Radiology, University of Missouri-Columbia, One Hospital Drive, Columbia, MO 65212.

IEEE Log Number 8927864. quency components in a pulse is obtained by applying the Taylor series expansion. The main drawback of the Taylor series is the requirement for a large number of terms in the expansion to provide an adequate representation of the field at points moderately far off axis or at frequencies significantly away from the reference frequency for a broad-band pulse [10].

An alternative algorithm for calculating the beam profile for a single element circular transducer is reported here. Although this algorithm is not as fast as the Taylor series expansion, it does not have the restrictions previously mentioned. It performs well in calculating the pressure everywhere in the field including the near field and points at large distances from the axis of the transducer.

\section{Representation of the Pressure Field}

To calculate the sinusoidal pressure at field point $Q$ of the transducer for some frequency $\omega$, a two-dimensional (2-D) integral is required:

$$
p(\dot{r}, \omega)=\iint_{S} \frac{e^{i k_{c}}\left|\dot{r}-\dot{r}^{\prime \prime}\right|}{\left|\dot{r}-\dot{r}^{\prime \prime}\right|} d s^{\prime \prime}
$$

where $d s^{\prime \prime}$ is the area element at position $\dot{r}^{\prime \prime}$ on the transducer surface (see Fig. 1.) and $S$ is the total radiating surface of the transducer. $k_{c}$ is the complex wavenumber, given by

$$
k_{c}=\omega / c(\omega)+i \alpha(\omega)=k+i \alpha(\omega)
$$

where $\omega$ is the angular frequency, $c(\omega)$ is the speed of sound, $\alpha(\omega)$ the attenuation coefficient and $k=\omega / c$, is the wave number.

For a disc-shaped or a circularly symmetric focused transducer, the double integral can be reduced to a single one [7] having the form

$$
p(\dot{r}, \omega)=\frac{2 A}{s}\left|\int_{C^{\prime}}^{D^{\prime}} \beta\left(r^{\prime}\right) e^{i k_{c} r^{\prime}} d r^{\prime}+\frac{F}{i k_{c}}\right|
$$

where $A$ is the radius of curvature of the radiating surface and $s$ is the distance from the center of curvature of the transducer to the field point $Q$.

In (3), $C^{\prime}$ is the distance from the field point $Q$ to the closest point on the edge of the disc transducer, and $D^{\prime}$ is the distance from the field point to the furthest point on 


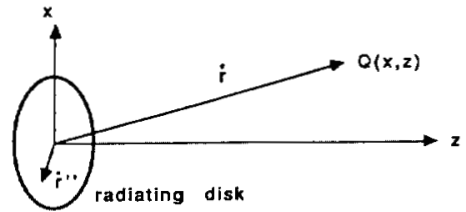

Fig. 1. Coordinates used for determining pressure at field point $Q$. Origin is at center of radiating disk, $z$-axis is along axis of symmetry of disk and $x$ is distance from $Q$ to $z$-axis. $r$ is vector from origin to field point and $r^{\prime \prime}$ is vector from origin to point on disk surface.

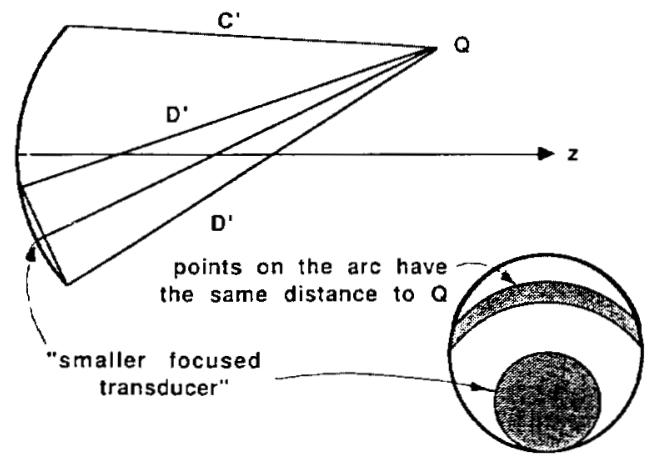

Fig. 2. When computing pressure at point $Q$, transducer is viewed as consisting of two parts. One part is smaller disk transducer whose axis of symmetry is through $Q$ and for which contribution to pressure is nonintegral term. Other part is divided into ares along which phase of wave contributing to pressure at point $Q$ is same. $C^{\prime}$ and $D^{\prime}$ are minimum and maximum distances from $Q$ to this part of transducer.

the edge, see Fig. 2. These distances are given by

$$
\begin{aligned}
& C^{\prime}=\left[\left(A^{2}+s^{2}+2 A s \cos (\Phi+\phi)\right]^{1 / 2}\right. \\
& D^{\prime}=\left[\left(A^{2}+s^{2}+2 A s \cos (\Phi-\phi)\right]^{1 / 2}\right.
\end{aligned}
$$

where the angles $\phi$ and $\Phi$ are seen in Fig. 3 .

$\beta\left(r^{\prime}\right)$ is essentially half of the angle of the arc on the transducer with the same distance $r^{\prime}$ to the field point $Q$. $\beta\left(r^{\prime}\right)$ is deduced in [7].

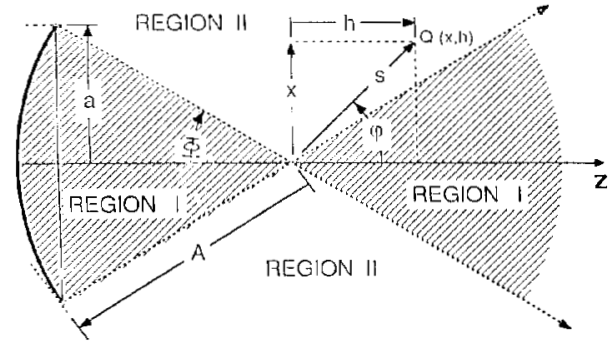

Fig. 3. Illustration of parameters used in (3)-(7) for computing pressure field for single element transducer (adapted from Timothy Hall's doctoral thesis).

This integrand is an enveloped sinusoidal function. The number of cycles in the integrand can be large, depending on the frequency and the difference between $C^{\prime}$ and $D^{\prime}$. However, the envelope of the sinusoidal function changes slowly as $r^{\prime}$ varies from $C^{\prime}$ to $D^{\prime}$. Its shape is mainly determined by $\beta\left(r^{\prime}\right)$ since the attenuation part only changes slightly in the small distance from $C^{\prime}$ to $D^{\prime}$. The actual shape of $\beta\left(r^{\prime}\right)$ depends on whether the field point is in Region I or Region II, shown in Fig. 3. In Region I, $\beta\left(r^{\prime}\right)$ monotonically changes from 0 to $\pi$ when $r^{\prime}$ goes from $C^{\prime}$ to $D^{\prime}$. An example is given in Fig. 4, which shows $\beta\left(r^{\prime}\right)$ as well as $\beta\left(r^{\prime}\right) \cos \left(k r^{\prime}\right)$ at the point $z=$ $40 \mathrm{~mm}, x=5 \mathrm{~mm}$, for a $3.5-\mathrm{MHz}, 18.8-\mathrm{mm}$ diameter transducer that has a radius of curvature of $10.97 \mathrm{~cm}$. (It is assumed that the medium has a speed of sound of 1561 $\mathrm{m} / \mathrm{s}$ at center frequency and an attenuation coefficient slope of $0.5 \mathrm{db} / \mathrm{cm}-\mathrm{MHz}$.) Fig. 5 gives the integrand for a field point at $z=40 \mathrm{~mm}$ and $x=10 \mathrm{~mm}$, for the same condition as the previous figure. This field point is in Region II, where $\beta\left(r^{\prime}\right)$ varies from 0 to a maximum value, then drops back to 0 . Notice that in this case the integrand has more than 10 cycles.

The basic idea of the fast algorithm reported in this paper is that the slowly changing envelopes in Figs. 4 and

$$
\beta\left(r^{\prime}\right)=\cos ^{-1}\left\{\frac{h+\sqrt{A^{2}-a^{2}}-\left(r^{\prime 2}+s^{2}-A^{2}\right) \cos \phi / 2 s}{(\sin \phi / 2 s) \cdot \sqrt{4 s^{2} r^{\prime 2}-\left(r^{\prime 2}+s^{2}-A^{2}\right)^{2}}}\right\}
$$

The quantity $F$ is given by

$$
F= \begin{cases}\pi \sigma \cdot\left\{e^{i k_{c}(A-s \sigma)}-\exp \left(i k_{c} \sqrt{ } A^{2}+s^{2}+2 A s \cos (\Phi-\phi \sigma)\right)\right\}, & \text { in region I of Fig. } 3 \\ 0, & \text { in region II of Fig. } 3\end{cases}
$$

where $\sigma=h /|h|$.

In (3), the transducer is viewed as consisting of two parts, as shown in Fig. 2. $F$ represents the contribution of a "smaller focused transducer" whose axis is through the field point. The integral accounts for the contribution from the rest of the transducer.

\section{The Fast Algorithm}

The most time consuming computational part of (3) is

$$
f\left(r^{\prime}, \omega\right)=\int_{C^{\prime}}^{D^{\prime}} \beta\left(r^{\prime}\right) e^{i k_{c} r^{\prime}} d r^{\prime} .
$$

5 can be expanded into a limited term polynomial, and thus the integral in (8) can be converted into a closed form.

We can rewrite the exponential in (8) as

$$
e^{-i k_{c} r^{\prime}}=e^{-\alpha(\omega) r^{\prime}}\left[\cos \left(k r^{\prime}\right)+i \sin \left(k r^{\prime}\right)\right] .
$$

Thus the envelope of the integrand is $\beta\left(r^{\prime}\right) e^{-\alpha(\omega) r^{\prime}} . \beta\left(r^{\prime}\right)$ is only a function of the field point location, while the attenuation part is a function of both the location and the frequency. We make an approximation that allows a separation of the attenuation term into a location dependent part and a frequency dependent part. The latter can then be pulled out of the integral. Expanded at the center fre- 


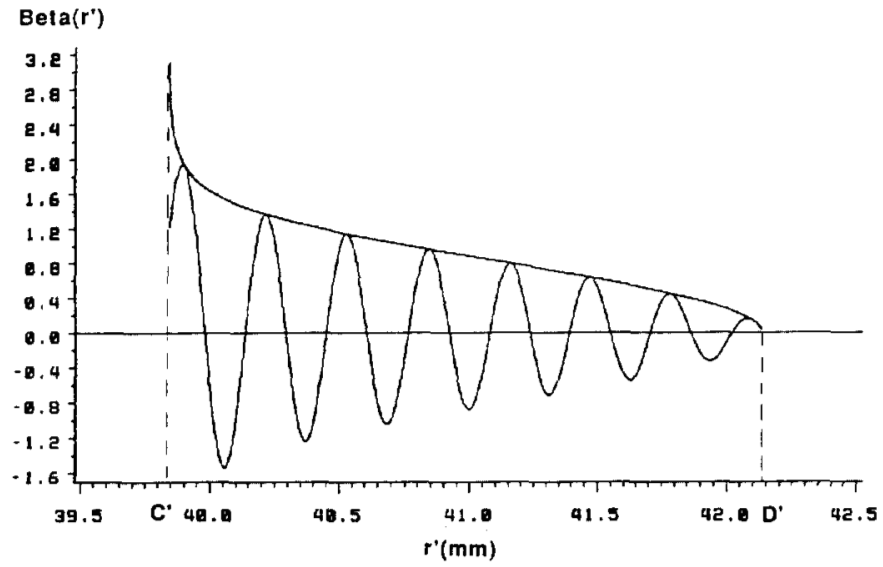

Fig. 4. Envelope function $\beta\left(r^{\prime}\right)$ versus dummy variable of integration, $r^{\prime}$, plotted for point in region I shown in Fig. 3. Here $\beta\left(r^{\prime}\right)$ changes monotonically from $\pi$ to 0 as $r^{\prime}$ varies from $C^{\prime}$ to $D^{\prime}$. This plot is for point at $x=5 \mathrm{~mm}$ and $z=40 \mathrm{~mm}$. $\beta\left(r^{\prime}\right) \cos \left(k r^{\prime}\right)$ for $\omega=3.5-\mathrm{MHz}$ is also shown.

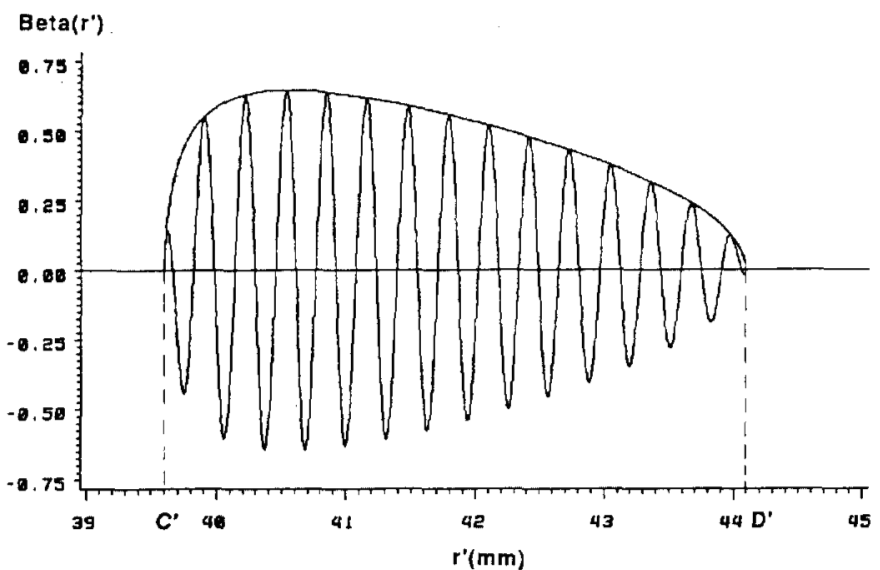

Fig. 5. $\beta(r)$ in region II changes from 0 to maximum value, then returns to 0 as $r^{\prime}$ varies from $C^{\prime}$ to $D^{\prime}$. This plot is for point at $x=10 \mathrm{~mm}$ and $z=40 \mathrm{~mm} . \beta\left(r^{\prime}\right) \cos \left(k r^{\prime}\right)$ for $\omega=3.5-\mathrm{MHz}$ is also plotted.

quency $\omega$, and mean distance $r_{0}$ as

$$
\alpha(\omega) \cdot r^{\prime} \approx \alpha(\omega) \cdot r_{0}+\alpha_{0} \cdot\left(r^{\prime}-r_{0}\right) .
$$

Here $\alpha_{0}=\alpha(\omega)$, is the attenuation coefficient at the center frequency $\omega_{0}$. Thus the attenuation $\alpha(\omega) \cdot r^{\prime}$ is represented as the sum of two parts. One part is the actual frequency dependent attenuation coefficient multiplied by a constant distance $r_{0}$, which is equal to the average of $C^{\prime}$ and $D^{\prime}$; the second part is the center frequency attenuation coefficient times the difference between $r^{\prime}$ and $r_{0}$. For a given field point any error due to this approximation can be made negligible by choice of integral parameters discussed in Section IV.

Equation (8) can then be written as

$$
\begin{aligned}
f\left(r^{\prime}, \omega\right)= & e^{-\alpha(\omega) r} \int_{C^{\prime}}^{D^{\prime}} \beta\left(r^{\prime}\right) e^{-\alpha_{0}\left(r^{\prime}-m\right)} \\
& \cdot\left[\cos \left(k r^{\prime}\right)+i \sin \left(k r^{\prime}\right)\right] d r^{\prime} .
\end{aligned}
$$

Let $I_{r}$ be the real part of the integrand in (11), i.e.,

$$
I_{r}=\int_{C^{\prime}}^{D^{\prime}} \beta\left(r^{\prime}\right) e^{-\alpha_{0}\left(r^{\prime}-r_{0}\right)} \cos \left(k r^{\prime}\right) d r^{\prime}
$$

The envelope may be represented by a polynomial of or$\operatorname{der} N$ as

$$
\beta\left(r^{\prime}\right) e^{-\alpha_{0}\left(r^{\prime}-r_{0}\right)}=\sum_{j=0}^{N} A_{j} \cdot r^{\prime j} .
$$

Equation (12) then becomes

$$
I_{r}=\sum_{j=0}^{N} A_{j} \int_{C^{\prime}}^{D^{\prime}} r^{\prime j} \cdot \cos \left(k r^{\prime}\right) d r^{\prime} .
$$

Similarly, the imaginary part, $I_{i}$ looks like

$$
I_{i}=\sum_{j=0}^{N} A_{j} \int_{C^{\prime}}^{D^{\prime}} r^{j} \cdot \sin \left(k r^{\prime}\right) d r^{\prime} .
$$

The integrals in (14) and (15) have closed forms. They can be computed recursively by the following formulas:

$$
\begin{aligned}
\int_{C^{\prime}}^{D^{\prime}} r^{\prime j} \cos \left(k r^{\prime}\right) d r^{\prime}= & \frac{r^{\prime j}}{k}\left[\sin \left(k D^{\prime}\right)-\sin \left(k C^{\prime}\right)\right] \\
& -\frac{j}{k} \int r^{i j}-1 \sin \left(k r^{\prime}\right) d r^{\prime} \\
\int_{C^{\prime}}^{D^{\prime}} r^{\prime j} \sin \left(k r^{\prime}\right) d r^{\prime}= & \frac{-r^{\prime j}}{k}\left[\cos \left(k D^{\prime}\right)-\cos \left(k C^{\prime}\right)\right] \\
& +\frac{r^{\prime}}{k} \int r^{\prime j-1} \cos \left(k r^{\prime}\right) d r^{\prime} .
\end{aligned}
$$

The total integral in (8) becomes

$$
f\left(r^{\prime}, \omega\right)=e^{-\alpha(\omega) r_{0}}\left(I_{r}+I_{i}\right)
$$

\section{Considerations in Implementation}

In programming (18), several steps have been taken to optimize the performance. First, we further divide the integral range $\left(C^{\prime}, D^{\prime}\right)$ into $m$ subranges as

$$
\begin{aligned}
\int_{C^{\prime}}^{D^{\prime}} f^{\prime} d r^{\prime}= & \int_{C^{\prime}}^{D_{1}} f^{\prime} d r^{\prime}+\int_{C_{2}}^{D_{2}} f^{\prime} d r^{\prime}+\cdots \\
& +\int_{C_{n}}^{D_{n}} f^{\prime} d r^{\prime}+\cdots+\int_{C_{m}}^{D^{\prime}} f^{\prime} d r^{\prime}
\end{aligned}
$$

where $C_{n+1}=D_{n}$, and $f^{\prime} d r^{\prime}$ represents the integrand of (17). This enables us to use a lower-order polynomial expansion and reduces the error for the attenuation approximation. The size of each subrange influences both the accuracy and the computation time. Since the integral range $d^{\prime}=D^{\prime}-C^{\prime}$ increases when the field point is further from the transducer axis, so does the number of subranges, $m$, if we want to keep the subrange size constant. Thus the computation time for the pressure at a point changes with location.

Secondly, we shift the integral range from $\left(C_{n}, D_{n}\right)$ to $(0, d)$, where $d$ is the size of the subrange, given by $d=$ 
$d^{\prime} / m$. This is done by a simple change of the variables $r^{\prime}$ to $r$ as

$$
\begin{aligned}
r & =r^{\prime}-C_{n} \\
d & =D_{n}-C_{n}
\end{aligned}
$$

This change greatly compresses the magnitude range of the polynomial and reduces computation errors.

The subrange size, $d$, and $N$ are correlated since a larger integral range needs a higher-order polynomial $N$, but a smaller number of subranges, $m$. For field points far away from the beam axis, the number of integral subranges can become large, consuming computation time proportionally with very limited improvement in accuracy. So, a limitation of the subrange number is set at $M$. When $m>$ $M, m$ is set equal to $M$, and a larger integral subrange $d$ is used.

These steps result in three computational parameters that must be selected:

1) The size of the integral subrange, $d$.

2) The order of the polynomial, $N$.

3) The maximum number of integral subranges, $M$.

We computed the pressure fields of the transducer described in Section V with different combinations of these parameters. Consider both accuracy and time savings, the combination of $N=6, d=0.25 \mathrm{~mm}$ and $M=10$ performs well and is used in the following examples. Thus, for each subrange, 6 different values for $r^{\prime}$ are used to generate simultaneous linear equations using (13). The $A_{i}$ values are then obtained and used in (16) and (17) to compute the pressure.

\section{Results}

All computations were done on a VAXstation II/GPX microcomputer and the programs were developed with Fortran 77.

\section{A. Lateral Beam Profile}

We compared the results from a 96-point GQ algorithm and the fast algorithm for computation of the field from a single element focused transducer. Calculations are done along a line perpendicular to the transducer axis, and the results are called "lateral beam profiles." For the results shown here, there are 50 points $/ \mathrm{cm}$ used to represent the profile. The transducer and the medium conditions are the same as that previously mentioned $(3.5-\mathrm{MHz}, 18.8-\mathrm{mm}$ diameter transducer that has a radius of curvature of 10.97 $\mathrm{cm}$; the medium has a speed of sound of $1561 \mathrm{~m} / \mathrm{s}$ and an attenuation coefficient slope of $0.5 \mathrm{db} / \mathrm{cm}-\mathrm{MHz}$ ).

Figs. 6(a) and (b) show plots of the pressure amplitude and phase vs. distance from the axis of the transducer at a depth of $10 \mathrm{~cm}$ and frequency of $3.5-\mathrm{MHz}$. Data are presented for both the fast algorithm and the Gaussian quadrature. Both algorithms give nearly identical results.

Figs. 7(a) and (b) show that the fast algorithm produces results that are very close to those obtained with the Gaussian quadrature even when the field point is far from

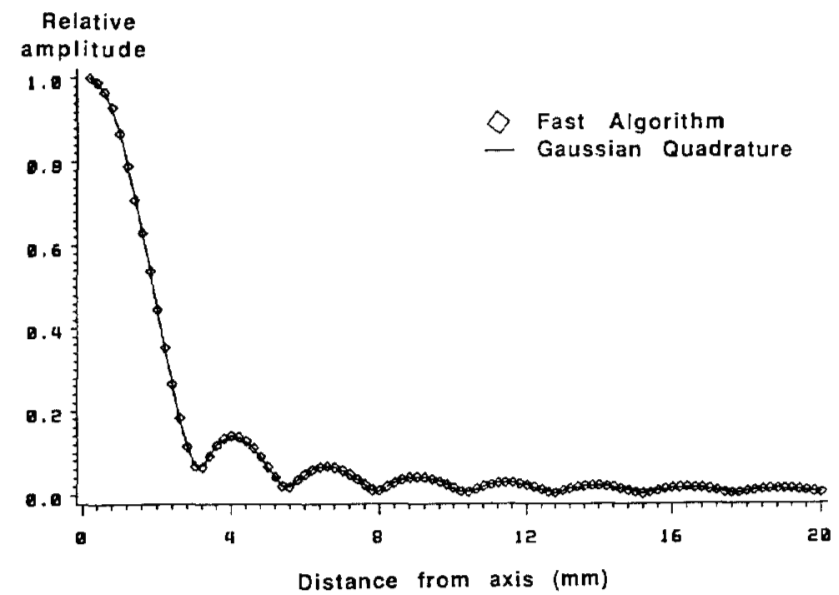

(a)

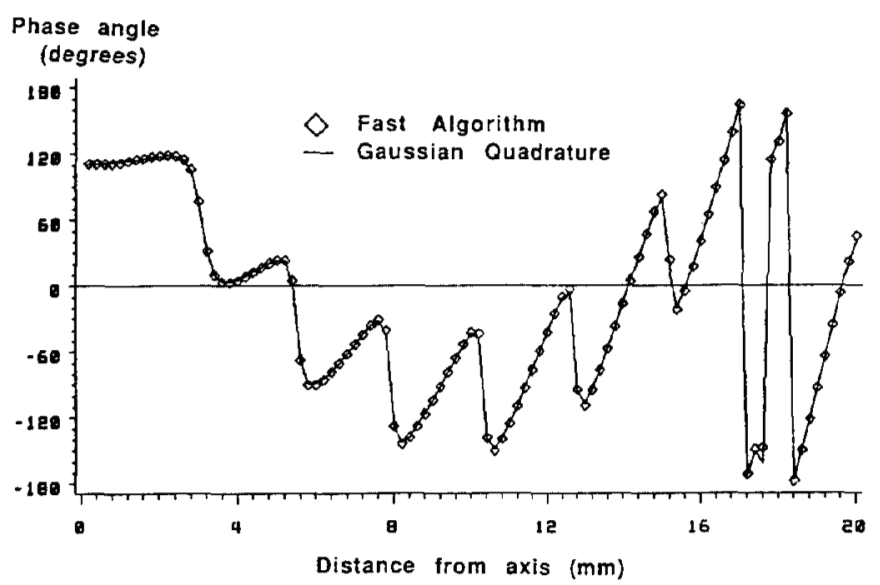

(b)

Fig. 6. Comparison between beam profiles computed using Fast Algorithm and using 96 point Gaussian Quadrature routine. (a) Relative pressure amplitude; (b) Phase angle. Calculations are done for $3.5-\mathrm{MHz} 18.8$ $\mathrm{mm}$ diameter, $10.97-\mathrm{cm}$ radius of curvature transducer insonifying medium whose speed of sound is $1561 \mathrm{~m} / \mathrm{s}$ at center frequency and whose attenuation coefficient is $0.5 \mathrm{db} / \mathrm{cm}-\mathrm{MHz}$. This profile is at $10 \mathrm{~cm}$ from transducer.

the transducer axis. Lateral beam profiles are shown out to a distance of $40 \mathrm{~mm}$ at a depth that is well within the near field of the transducer. In this example the data are plotted on a logarithmic scale so that a comparison could be made with results of the GQ algorithm at very low relative amplitude levels.

\section{B. Signals from Volumetric Scatterers}

When calculating the echo signal from a volume containing scatterers [2] we take advantage of the fact that the beam profile of a disk transducer is symmetric about its axis. Each pressure data point actually determines the pressure within a ring centered about the axis. The relative contribution to the echo signal from each ring is given by the pressure amplitude squared times the ring diameter.

To test the usefulness of the fast algorithm for these computations we have computed the associated error, defined as the difference between results obtained with it and with a 96-point GQ integration routine. Let $\operatorname{amp}(j, \omega)$ be the pressure amplitude obtained with the fast algorithm 


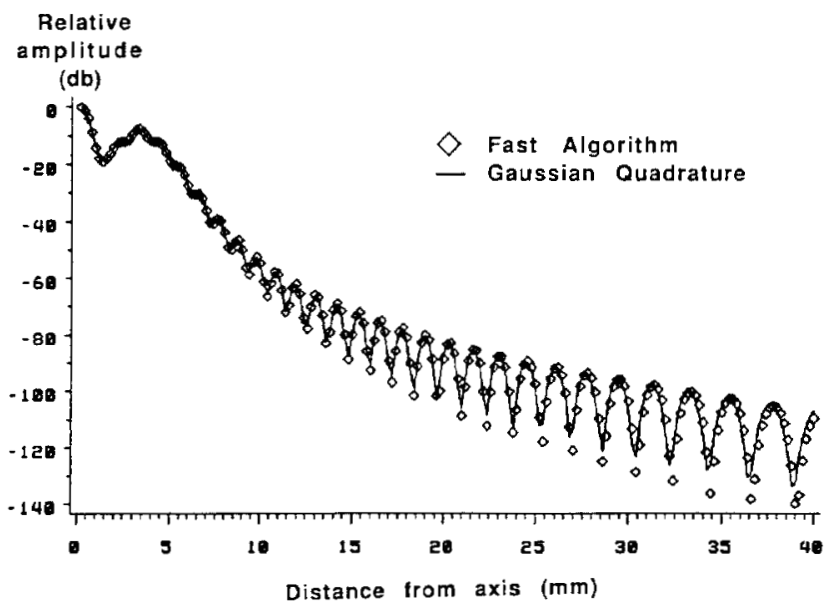

(a)

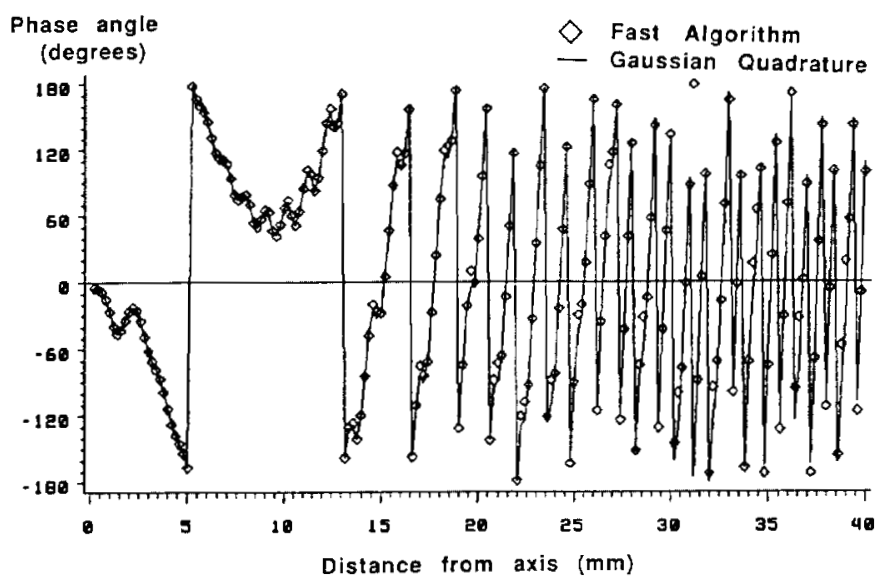

(b)

Fig. 7. Comparison between beam profiles computed using Fast Algorithm and using 96 point Gaussian Quadrature routine. (a) Relative pressure amplitude (note logarithmic scale is used to show correspondence over very large range of amplitudes); (b) Phase angle. Same transducer and medium are used as in case of Fig. 6 . This profile is at $4 \mathrm{~cm}$ from transducer.

and $\operatorname{amp} o(j, \omega)$ be the amplitude obtained with the GQ at frequency $\omega . j$ refers to the point in the lateral profile, where the distance between points is $b$. Similarly let angl $(j, \omega)$ be the phase angle obtained using the fast algorithm and anglo $(j, \omega)$ be the phase angle obtained using the GQ.

The square of amplitude error is defined as

$$
\frac{\sum_{j=1}^{K}[\operatorname{amp}(j, \omega)-\operatorname{ampo}(j, \omega)]^{2}(b \cdot j)^{2}}{\sum_{j=1}^{K} \operatorname{ampo}^{2}(j, \omega)(b \cdot j)^{2}} .
$$

The phase error is defined as

$$
\frac{1}{K} \sum_{j=1}^{K}|\operatorname{angl}(j, \omega)-\operatorname{anglo}(j, \omega)| .
$$

where $K$ is the number of points calculated in the radial direction of the beam profile.

Tables I and II present results of this test for a typical transducer for three different ultrasound frequencies. The
TABLE I

Comparison of Reslizts of Lateral Beam Profile Computations using the Fast Algorithm and USing a 96-point Gaussian Quadrature ALGORITHM

\begin{tabular}{cccc}
\hline \hline $\begin{array}{c}\text { Depth } \\
(\mathrm{cm})\end{array}$ & $\begin{array}{c}\text { Frequency } \\
\text { (MHz) }\end{array}$ & $\begin{array}{c}\text { Amplitude } \\
\text { Error } \\
\text { (percent) }\end{array}$ & $\begin{array}{c}\text { Phase Angle } \\
\text { Error } \\
\text { (degree) }\end{array}$ \\
\hline 4 & 1.5 & 0.545 & 0.436 \\
& 3.5 & 1.356 & 1.387 \\
& 5.5 & 2.380 & 2.560 \\
Computation time for GQ 669.33 s & & \\
& for Fast $94.84 \mathrm{~s}, 1 / 7.54$ of GQ & \\
11 & 1.5 & 0.794 & 2.330 \\
& 3.5 & 2.080 & 4.450
\end{tabular}

Computation time for GQ $646.90 \mathrm{~s}$

for Fast 71.22 s, $1 / 9.0831$ of $\mathrm{GQ}$

$18.8-\mathrm{mm}$ diameter, $11-\mathrm{cm}$ radius of curvature transducer; 100 points, $2-\mathrm{cm}$ off axis.

TABLE II

Comparison of Results of Lateral Beam Profile Computations using the Fast Algorithm and USING a 96-POINT Gaussian QUadrature AlgORITHM

\begin{tabular}{cccc}
\hline $\begin{array}{c}\text { Depth } \\
(\mathrm{cm})\end{array}$ & $\begin{array}{c}\text { Frequency } \\
(\mathrm{MHz})\end{array}$ & $\begin{array}{c}\text { Amplitude } \\
\text { Error } \\
\text { (percent) }\end{array}$ & $\begin{array}{c}\text { Phase Angl } \\
\text { Error } \\
\text { (degree) }\end{array}$ \\
\hline 4 & 1.5 & 0.174 & 0.122 \\
& 3.5 & 0.450 & 0.294 \\
& 5.5 & 0.906 & 0.451
\end{tabular}

Computation time for GQ $335.64 \mathrm{~s}$ for Fast $43.88 \mathrm{~s}, 1 / 7.65$ of $\mathrm{GQ}$

$\begin{array}{rrr}11.5 & 0.801 & 0.122 \\ 3.5 & 1.810 & 1.460 \\ 5.5 & 3.180 & 3.640\end{array}$

Computation time for G.Q. $321.48 \mathrm{~s}$ for Fast $23.65 \mathrm{~s}, 1 / 13.65$ of $\mathrm{GQ}$

18.8-mm diamter, $11-\mathrm{cm}$ radius of curvature transducer; 50 points, $1 \mathrm{~cm}$ off axis.

percent error when using the fast algorithm is lower for lower frequencies and for situations where the beam profile does not have to be computed far from the transducer axis. Division into even larger numbers of subranges would decrease the percent error, but at the expense of longer computation times.

Also shown in the tables are the computation times needed for both routines. We can see that the time saving is more when the field points are closer to the axis or further from the transducer. Near the radius of curvature of the transducer the fast algorithm is nine times faster than the Gaussian quadrature routine out to $2-\mathrm{cm}$ off axis and 13 times faster if the profile is only $1 \mathrm{~cm}$ off axis.

\section{Discussion}

We have also compared results of backscatter coefficient determinations [3] when the fast algorithm is utilized to compute pressure fields to results obtained using a GQ routine for pressure field computations. Assuming 
an $18.3-\mathrm{mm}$ diameter, $13.7-\mathrm{cm}$ radius of curvature transducer and a phantom with tissue-like attenuation, results obtained with the fast algorithm were within 0.7 percent of those obtained using the GQ when the scattering volume was any where in the range of $7-13.6 \mathrm{~cm}$ depth. The computation time for the fast algorithm was less than $1 / 10$ of that for the GQ. Other depth ranges and transducer geometries produce similar results.

The algorithm described here is not necessarily as fast as a Taylor series representation for pressure beam computations, also developed in our laboratory, particularly when sampling densely in the frequency domain. However, the fast algorithm is not subject to the type of transaction errors that the Taylor series representation is [10]. Consequently, both routines would appear to have advantages. For example, the Taylor series seems particular well suited for situations where the scattering volume is near the center of curvature of the transducer or where the range of frequencies is limited. The fast algorithm might be advantageous as a general routine or as a routine to utilize prior to adapting the Taylor series.

In implementing the fast algorithm, the parameters $(N$, $d, M$ ) influence the results (accuracy and time) in many ways. Different field locations need different parameters to get the best results. Studies could be done to develop an algorithm to assign sets of parameters for different locations or different accuracy or computation time constraints.

\section{Conclusion}

We have developed a workable fast algorithm to compute the ultrasound pressure field from a circular, single element transducer. The algorithm is much faster than direct numerical methods currently in use. Lateral beam profiles computed using the fast algorithm are nearly identical to profiles computed using a 96 -point GQ routine.

\section{ACKNOWLEDGMENT}

The authors are grateful to Dr. Timothy Hall for helpful discussions.

\section{REFERENCES}

[1] J. A. Zagzebski, E. L. Madsen, and M. M. Goodsitt, "Quantitative tests of a three-dimensional gray scale texture model," Ultrasonic Imaging., vol. 7, pp. 252-263, 1985.

[2] E. L. Madsen, M. F. Insana, and J. A. Zagzebski, "Method of data reduction for accurate determination of acoustic backscatter coefficients," J. Acoust. Soc. Am., vol. 76, no. 3, pp. 913-923, 1984.

[3] E. J. Boote, J. A. Zagzebski, E. L. Madsen, and T. J. Hall, "Instrument-independent acoustic backscatter coefficient imaging," Ultrason. Imaging, vol. 10, no. 2, pp. 121-138, 1988.

[4] G. R. Harris, "Review of transient field theory for a baffled planar piston," J. Acous. Soc. Am., vol. 70, no. 1, pp. 10-20, 1981.
[5] H. T. O'Neil, "Theory of focusing radiator," J. Acous. Soc. Am., vol. 21, pp. 516-526, 1949.

[6] A. Penttinnen and M. Luukkala, "The impulse response and pressure nearfield of a curved ultrasonic radiator," J. of Phys. D: Appl. Phys., vol. 9, pp. 1547-1557, 1976

[7] E. L. Madsen, M. M. Goodsitt, and J. L. Zagzebski, "Continuous waves generated by focused radiators," J. Acous. Soc. Am., vol. 70, no. 5, pp. 1508-1517, 1981.

[8] M. M. Goodsitt, E. L. Madsen, and J. A. Zagzebski, "Field patterns of pulsed focused ultrasonic radiations in attenuation and nonattenuating media," J. Acous. Soc. Am., vol. 71, pp. 318-329, 1982.

[9] E. L. Madsen, T. J. Hall, J. A. Zagzebski, and M. F. Insana, "Use of Taylor series expansions for time savings in computation of accurate transducer pressure fields," IEEE Trans. Ultrason. Ferroelect. Freq. Contr., vol. UFFC-34, pp. 301-308, 1987

[10] T. J. Hall, E. L. Madsen, and J. A. Zagzebski, “A Taylor series expansions for time savings in accurate computation of focused ultrasound pressure fields," Ultrason. Imaging, vol. 9, pp. 203-219, 1987.

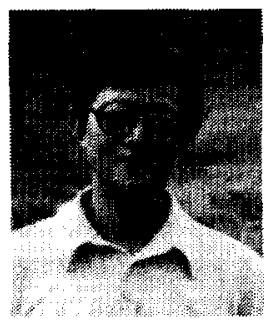

Lin Xin Yao received the M.S. degree in electronics in 1981 from Fudan University, Shanghai, China, and the M.S. degree in medical physics in 1988 from the University of Wisconsin, Madison.

From 1981 to 1984 he was a faculty member in the electrical department at Anhui Institute of Mechanical and Electrical Technology, Wuhu, China. In 1984 he came to the electrical and computer engineering department at the University of Wisconsin, Madison as a Visiting Scholar. Currently, he is in the Medical Physics Department at University of Wisconsin, Madison pursuing the Ph.D. degree. He is interested in tissue characterization, lesion detectability and quality control in medical ultrasound.

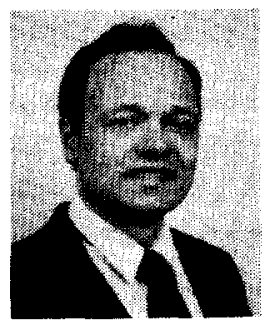

James A. Zagzebski received the B.S. degree in physics from St. Mary College, Winona, MN, and the M.S. degree in physics and the Ph.D. degree in radiological science from the University of Wisconsin, Madison, in 1968 and 1972, respectively.

$\mathrm{He}$ is Professor of Medical Physics and of Radiology and Human Oncology at the University of Wisconsin, Madison. His research interests include medical imaging, diagnostic ultrasound, and ultrasound hyperthermia.

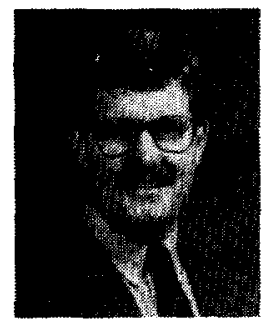

Evan J. Boote was born in Hull, IA, in 1961. He received the B.S. degree in physics from Hope College, Holland, MI, in 1983, the M.S. and Ph.D. degrees in medical physics from the University of Wisconsin, Madison, in 1985 and 1988, respectively.

From 1984 to 1988, he was involved in research at the University of Wisconsin. This research was aimed at diagnostic ultrasound quality assurance and ultrasonic tissue characterization. Currently, he is Assistant Professor of Radiology at the University of Missouri Columbia Medical School. 